\title{
Enhanced oral hygiene interventions as a risk mitigation strategy for the prevention of non-ventilator-associated pneumonia: a systematic review and meta-analysis
}

\author{
Poolakkad S. Satheeshkumar, ${ }^{* 1,2}$ Stefania Papatheodorou ${ }^{3}$ and Stephen Sonis ${ }^{2,4}$
}

\section{Key points}

Ongoing professional dental care is the most predictably effective preventive intervention for non-ventilated pneumonia (NVAP) for patients in long-term care facilities.
Structured oral care may be warranted for hospitalised patients at risk of NVAP.
Additional, prospective, randomised, controlled clinical trials are needed to validate the utility of ora care interventions as a preventive strategy for NVAP.

\begin{abstract}
Background Healthcare-acquired pneumonias are a significant risk for nursing home and hospital patients. While oral care interventions (OCIs) have been found to be effective in reducing the risk of ventilator-associated pneumonia (VAP), their utility in mitigating non-ventilator-associated pneumonias (NVAP) remains unknown. We performed a structured meta-analysis of randomised and non-randomised clinical trials of enhanced oral hygiene procedures on NVAP.
\end{abstract}

Methods We searched PubMed and Embase to include clinical trials (randomised and non-randomised), and observational (retrospective and prospective) and quasi-experimental studies examining the effect of any method of OCI on incidence of NVAP.

Results After quality assessment and consensus agreement between authors, we synthesised six randomised clinical trials (3,891 patients), two non-randomised trials (2,993 patients), and separately assessed a retrospective trial (143 patients) and a quasi-experimental study (83 patients). Most studies, performed in nursing homes, did not show a significant association between $\mathrm{OCl}$ and NVAP prevention (RR random $0.89,95 \% \mathrm{Cl} 0.64-1.25, \mathrm{p}$ value 0.50 ). Likewise, the non-randomised trials failed to show an association between NVAP risk and OCI (RR random 1.42, 95\% Cl, 0.70-2.88, p value 0.32). However, in the subgroup analysis comparing dental professional involvement in care vs usual care, reduced NVAP risk was demonstrated (RR random $0.65,95 \% \mathrm{Cl} 0.43-0.98, \mathrm{p}$ value 0.03$)$.

Conclusions Study results suggest that professional dental care may confer some benefit among NVAP patients. The lack of consistent $\mathrm{OCl}$ protocols, data in hospitalised patients and robust randomised clinical trials do not allow definitive conclusions about the contribution of OCI in mitigating NVAP risk.

\section{Introduction}

Pneumonias acquired in acute and chronic healthcare facilities are a significant risk for patients. A 2015 survey reported that hospitalacquired infections affect approximately $3.2 \%$ of patients hospitalised in the United States, ${ }^{1}$ or $1,184,000$ cases this year, at costs exceeding $\$ 40,000,000,000$. Among

${ }^{1}$ Harvard Medical School, Boston MA, USA; ${ }^{2}$ Primary Endpoint Solutions, Watertown MA, USA; ${ }^{3}$ Harvard School of Public Health, Boston MA, USA; "Brigham and Women's Hospital, Boston, MA, USA.

${ }^{*}$ Correspondence to: Poolakkad Satheeshkumar

Email: Satheeshkumar_PoolakkadSankaran@hms.harvard. edu

Refereed Paper.

Accepted 10 February 2020

https://doi.org/10.1038/s41415-020-1452-7 hospital-acquired infections, pneumonias are the most common with an overall incidence of $21.8 \% .^{2,3,4}$ Pneumonia is also clinically the most significant infection among the approximately 1.7 million nursing home patients in the United States, with an incidence of 0.3 to 2.3 episodes per 1,000 resident care days. ${ }^{5}$

Pneumonias in these populations are typically categorised based on their association with ventilator use. Ventilator-associated pneumonias (VAPs) have been best studied and protocols have been developed which have lowered their risk. In contrast, the prevalence of non-ventilatorassociated pneumonias (NVAPs) has remained essentially unchanged. ${ }^{1}$ Classic hospital-acquired pneumonias are defined as those that develop after 48 hours of hospital admission. ${ }^{6}$ While the definition for NVAPs can also be applied to nursing home patients, there are marked differences between the two populations, including length of stay (13.7 months for nursing homes $^{7}$ vs 6.1 days for acute care hospitals), ${ }^{8}$ demographics and co-morbidities. Nonetheless, given the potential importance of the oral cavity as a bacterial source for NVAP, we generally included both populations in the analysis, but analysed them separately.

The physiologic and healthcare costs of NVAPs are significant and have been welldescribed. ${ }^{2,48}$ The microbial aetiology of NVAP has been ascribed to pathogens associated with the upper aerodigestive tract, for which four potential routes of contamination have been hypothesised: aspiration of oropharyngeal secretions, food or gastric contents; inhalation of infectious aerosols; contiguous spread of infection; or haematogenous spread from nonpulmonary sources to the lung. ${ }^{9}$ The primary 
source of pathogens of pulmonary infections is suggested to be associated with aspiration of colonised secretions from the oropharynx. However, given the bacterial spectrum reported for NVAP, it is impossible to ignore the nose, nasopharynx or sinuses as also being important. A relationship between NVAP risk and dentate state is unresolved. ${ }^{10}$

Intensive oral care interventions (OCIs), regimens that reduce the oral cavity bacterial load, have been suggested to be effective in mitigating NVAP risk. The individual elements comprising these regimens have not been consistent and range dramatically in their intensity. However, trends in outcomes potentially support their utility. If professionally delivered oral care regimens are to be considered for universal standard of care for NVAP prevention, several critical questions require answers:

1. Is the nursing time, effort and instrumentation needed for an expanded oral hygiene programme justified by a cost/ benefit analysis; that is, how effective are expanded oral hygiene programmes in modifying risk of NVAP?

2. Are there specific risk factors which can prospectively identify patients at risk of NVAP and how do these patients specifically respond to oral hygiene programmes?

3. When is the optimum time to initiate OCIs; that is, are programmes which commence at the time of admission effective, or should oral hygiene programmes begin earlier, and if so, how much earlier?

4. Are the pathogens observed in NVAP found in the oral biofilm, where are the primary depots of pathogens and which OCIs best target those depots?

As a first step, we performed a structured meta-analysis in which we assessed randomised and non-randomised clinical trials, and observational studies that investigated the relative efficacy of enhanced OCI programmes on NVAP.

\section{Methods}

Search strategy and inclusion criteria

Using the Preferred Reporting Items for Systematic Reviews and Meta-Analyses (PRISMA) statement, ${ }^{11}$ a systematic literature appraisal was performed. The literature search was done in PubMed (inception until January 2019) and Embase (1990 to January 2019) using inclusive search terms (see Appendix 1). The searches included all study designs: clinical trials (randomised and non-randomised), observational studies (retrospective and prospective) and quasiexperimental studies. Title and abstracts were independently screened by two investigators (KS and SS) and disagreements regarding eligibility were discussed. Cross-referencing and supplementary literature searches were performed to examine references in topicrelated previously published reviews, and by manually searching bibliographies of the included articles and similar articles. Full-text screening of selected publications was done by two examiners and discrepancies were resolved by discussion. For each selected study, the study characteristics were extracted by two assessors which were then corroborated by a third researcher.

\section{Inclusion criteria}

Experimental and observational studies were included based on the following criteria:

1. Reported NVAP as a primary outcome

2. Conducted on hospitalised/chronic care facility adults $>18$ years of age who were not diagnosed as having pneumonia at the time of admission

3. Intervention or exposure to enhanced oral care, whether matched with placebo, usual care or comparable medication for preventing NVAP

4. Provided data in the form of point estimates and measure of $95 \%$ confidence intervals (CIs), or the data were required to be available to calculate those measures.

Studies were excluded if they did not provide specific NVAP results, as were those that used the term 'hospital-acquired pneumonia (HAP)' but did not differentiate NVAP from VAP. We also excluded the studies which were not published in English and those with results published as abstracts or poster presentations. If data from the same patient cohort was published more than once, we only included the study which most informed our outcome.

\section{Data extraction}

Data were extracted from eligible studies, independently, using pre-specified data extraction forms. For each included record, study characteristics were recorded by two independent members of the team and discrepancies were resolved by discussion. Characteristics included methods, country, setting, duration of follow-up, sample size, number of patients randomised, number of patients evaluated, inclusion and exclusion criteria, diagnosis of NVAP, intervention (type, dose and frequency of oral care), control (type, dose and frequency of oral care), outcome measures involving incidence of NVAP secondary endpoints, and funding source.

\section{Quality of studies}

Reviewers independently extracted and assessed the risk of bias for randomised controlled trials (RCTs), the random sequence generation (selection bias), allocation concealment (selection bias), blinding of participants and personnel (performance bias), blinding of outcome assessment (detection bias), incomplete outcome data (attrition bias), selective reporting (reporting bias) and other bias (for example, funding bias).

The quality of RCTs was assessed using the 'Risk of Bias' tool from the Cochrane Collaboration and the quality of observational studies was assessed using the NewcastleOttawa Scale (NOS). ${ }^{12,13}$ Case definition met the selection/outcome criteria if recorded in health services/study databases as actual diagnoses and did not meet the NOS criteria if self-reported and/or gathered by questionnaire. A similar approach was taken with ascertainment of enhanced oral care to meet relevant NOS criteria if recorded as prescriptions in health services/study databases and did not meet NOS criteria if self-reported and/or gathered by a non-validated questionnaire. For lost to follow-up, we considered any study with $\leq 10 \%$ lost to follow-up adequate. The remaining NOS criteria were followed routinely.

\section{Subgroup analyses}

We performed three subgroup analyses. In the first, we evaluated the effectiveness of chemical disinfection on NVAP risk, in the second, we compared the differences in effectiveness between enhanced oral hygiene regimens in which a dental profession (dentist/dental hygienist) vs. those which were administered solely by non-dentally qualified individuals and in the third, we evaluated the effectiveness of the enhanced oral hygiene regimens on the outcome of mortality due to NVAP.

\section{Statistical analysis}

Statistical analyses were based on comparing rates of total NVAP events between the enhanced OCI group and the control group. If the studies did not report the number of NVAP events and/or the total number of 
participants in the enhanced OCI group and the control group, we used RR, OR and a measure of variance $95 \%$ CIs to produce summary relative risk estimates and measure of variance 95\% CIs. Due to the expected clinical heterogeneity between studies, we decided a priori to use a DerSimonian and Laird (DL) random effects model for all analyses. ${ }^{14}$ Testing for heterogeneity between the studies was performed using Cochran's $\mathrm{Q}$ test ${ }^{15}$ and the $\mathrm{I}^{2}$ test. ${ }^{13}$ A p value $<0.05$ or an $\mathrm{I}^{2}$ higher than $50 \%$ were considered significant evidence for heterogeneity. Additionally, we used the Hartung-Knapp-Sidik-Jonkman (HKSJ) method $^{16}$ to retrieve more adequate error rates. Simulations have shown that the HKSJ method performs better than DL, especially when there is heterogeneity and the number of studies in the meta-analysis is small. ${ }^{16,17,18,19,20}$ Subgroup analysis was performed to assess whether there were differences between professional dental care and usual care, use of antimicrobial chlorhexidine (CHX) and usual care, and mortality due to pneumonia in enhanced OCI versus usual care. All statistical analyses were performed using RStudio, Version 1.1.456 (RStudio: Integrated Development for RStudio; RStudio, Inc., Boston, MA URL).

Visual assessment using Funnel's and Egger's plots for publication bias was not performed as we had less than ten studies included in the meta-analysis.

\section{Results}

Our initial search provided 16,611 records; duplicates of 6,223 were removed with the EndNote software X9.1.1 version. After title and abstract screen, 5,921 unrelated records were excluded, culminating in 302 records that were assessed for eligibility using full-text screening. Eleven studies were identified as meeting our inclusion criteria. Our search strategy led to identification of seven clinical trials, two non-randomised clinical trials, one quasi-randomised and one retrospective cohort study (Fig. 1).

Characteristics of the included studies are listed in Table 1 (see online supplementary information for further detail). Seven $\mathrm{RCTs}^{21,22,23,24,25,26,27}$ are included in our analysis, of which five $e^{21,22,23,26,27}$ were conducted in nursing homes, one in a stroke rehabilitation unit, ${ }^{24}$ and one in an intensive care unit (ICU). ${ }^{25}$ One study was not included in the qualitative assessment because of non-estimable risks, both in the intervention and the control group. ${ }^{24} \mathrm{~A}$ total

Fig. 1 Flow diagram showing summary of literature search and study selection

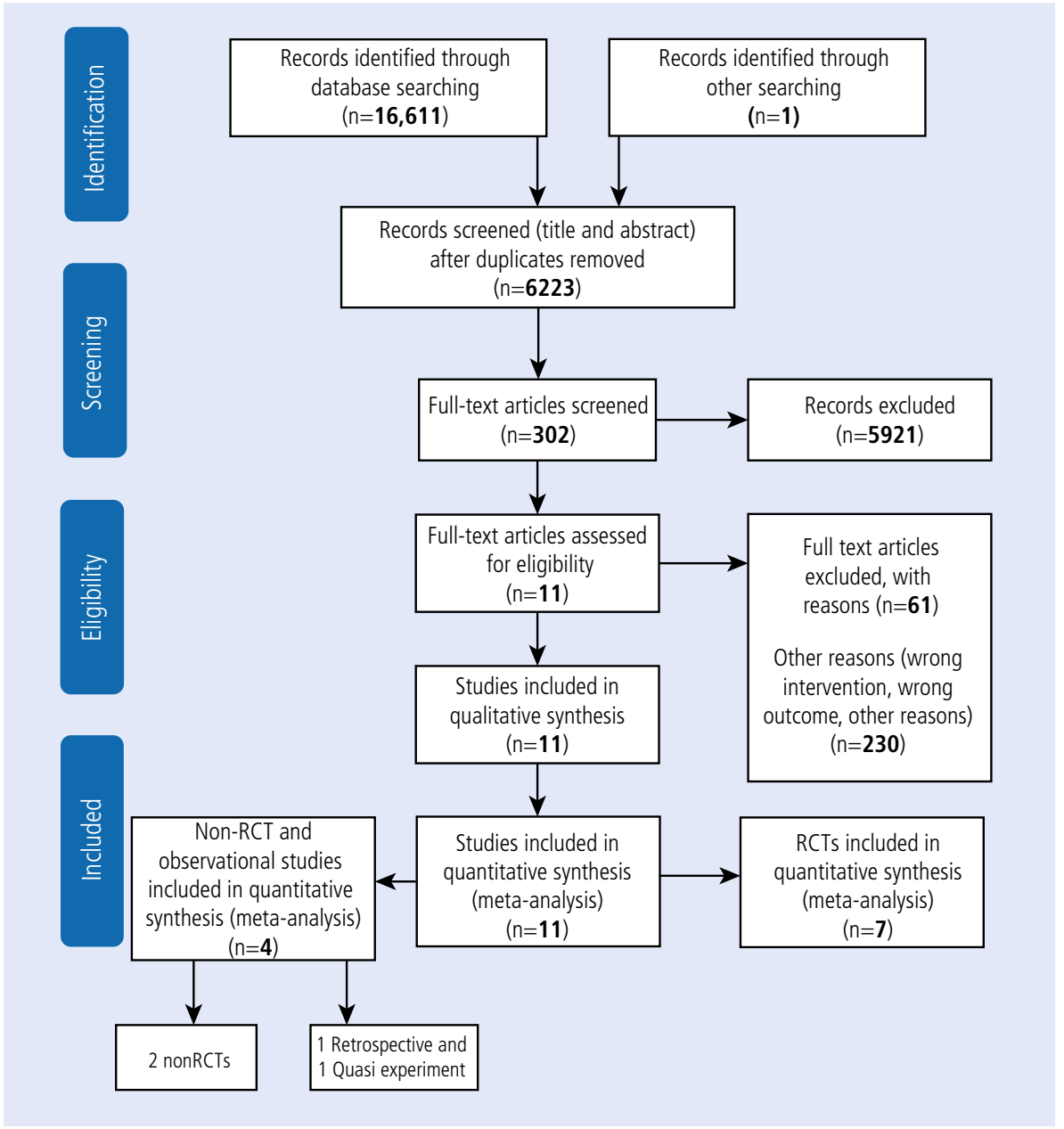

of 3,891 patients were included in the overall analysis. Among the non-RCTs, ${ }^{28,29}$ one study (number analysed: 2,890 ) was done in a nonintensive acute care hospital setting, ${ }^{28}$ and the other in nursing home residents. ${ }^{29}$ Among the other experiment designs, one study was a quasi-experimental trial (number analysed: 83 ) in a neurosurgical population outside the critical care environment, ${ }^{30}$ and the other study was a retrospective analysis (number analysed: 143) conducted in nursing home residents. ${ }^{31}$ We employed a per-protocol analysis (PPA) to understand the superior effects of treatment, as PPA provides an estimate of the true efficacy of an intervention, also recognising that PPA interpretation to actual practice may be confounded by an overstated treatment weight. ${ }^{32}$

\section{Meta-analysis of enhanced oral care in preventing NVAP - RCTs}

The meta-analysis of the six RCTs was performed using the DL and HSKJ methods. The DL method demonstrated a pooled relative risk of 0.89 (95\% CI: $0.64-1.25, \mathrm{p}$ value $=0.50, \mathrm{I}^{2}=65.2 \%, \mathrm{p}$ value $_{\text {het }}=0.01$, $\operatorname{tau}^{2}=0.08$ ) (Table 2). HKSJ adjustment of the CIs provided similar results. Since fewer than ten studies were included in the quantitative synthesis, publication bias assessment was not performed.

\section{Effect of oral CHX in the prevention of NVAP}

Subgroup analysis was performed to assess the effect of oral CHX rinsing on the prevention of NVAP ( $\mathrm{n}=3$ studies). Using the DL method, the combined effect size was $1.05(95 \%$ CI, 0.69 to 1.60 , $\mathrm{p}$ value $=0.80 ; \mathrm{I}^{2}=76.6 \%$, $\operatorname{tau}^{2}=0.08$ with $\mathrm{p}$ value $_{\text {het }}=0.01$, suggesting that the addition of $\mathrm{CHX}$ to an enhanced oral care regimen was ineffective in preventing NVAP (Table 3).

\section{Dental professional involvement in enhanced oral care in prevention of pneumonia}

Subgroup analysis of the impact of dental professional involvement (dentist or hygienist) in enhanced oral care versus usual oral care in the prevention of NVAP ( $\mathrm{n}=3$ studies) revealed 
a pooled relative risk of 0.65 using the $\mathrm{DL}$ method ( $95 \%$ CI, 0.43 to 0.98 , $\mathrm{p}$ value $=0.03$, $\mathrm{I}^{2}=0 \%, \operatorname{tau}^{2}=0$ with $\mathrm{p}$ value $\left._{\text {het }}=0.9\right)$. It appeared that oral care in which a dental professional was involved favourably reduced NVAP risk (risk reduction of 35\%) (Table 4).

Effects of enhanced oral care in the prevention of mortality due to pneumonia There was no impact of enhanced oral care in reducing NVAP-related mortality $(\mathrm{n}=4$ studies); pooled relative was $0.80(95 \%$ $\mathrm{CI}, 0.40$ to 1.63$), \mathrm{p}$ value $=0.54, \mathrm{I}^{2}=83 \%$, $\operatorname{tau}^{2}=0.38$ with $\mathrm{p}$ value $\mathrm{h}_{\text {het }}=0.00$ based on the DL method (Table 5).
Non-randomised clinical trials

Assessment of the two non-randomised clinical trials performed with DL method showed the pooled relative risk 1.42 (95\% CI, 0.70 to 2.88 ) $\mathrm{p}$ value $=0.32, \mathrm{I}^{2}=74 \%, \operatorname{tau}^{2}=0.19$ with $\mathrm{p}$ value $_{\text {het }}=0.05$ (Table 6).

Other studies

Two additional studies were included in the meta-analysis: a retrospective and a quasiexperiment study. The retrospective analysis noted an odds ratio of 1.21 (95\% CI 0.99-1.48), with a $\mathrm{p}$ value of 0.6 . The quasi-experimental study showed relative risk of 0.25 (95\% CIs $0.06,1.02) \mathrm{p}$ value of 0.05 .

\section{Discussion}

The oral cavity is a documented source of pathogens which might contribute to NVAP risk. Consequently, enhanced oral hygiene regimens aimed at reducing the oral bacterial load have been proposed as a risk mitigation strategy. While standard patientperformed oral hygiene is an integral part of a proactive health maintenance routine, definitive evidence of the health and cost benefits of more aggressive regimens on NVAP risk is critical for making the case for widening its implementation. Our aim was to assess clinical trial outcomes in this space

Table 1 Characteristics of the individual studies

\begin{tabular}{|c|c|c|c|c|c|c|}
\hline Reference (year) & Country & Study setting & Duration & $\begin{array}{l}\text { Number of } \\
\text { participants } \\
\text { evaluated }\end{array}$ & Intervention type & Control \\
\hline \multicolumn{7}{|c|}{ Randomised controlled trials } \\
\hline Adachi (2002) & Japan & Nursing homes & 24 months & 88 & Professional care & Usual oral care \\
\hline Bourigault (2011) & France & Nursing homes & 18 months & 2,513 & $\mathrm{CHX}$ oral rinse and enhanced oral care & Usual oral care \\
\hline Ohsawa (2003) & Japan & Nursing homes & 24 months & 49 & Professional care with povidone-iodine oral rinse & Usual oral care \\
\hline $\operatorname{Lam}(2013)$ & Hong Kong & Stroke rehabilitation unit & 31 months & 81 & Professional care with $\mathrm{CHX}$ oral rinse & $\begin{array}{l}\text { Oral hygiene } \\
\text { instructions }\end{array}$ \\
\hline Panchabhai (2009) & India & $\begin{array}{l}\text { ICU (medical and } \\
\text { surgical) }\end{array}$ & 8 months & 300 & $\mathrm{CHX}$ and usual care & $\begin{array}{l}\text { Potassium } \\
\text { permanganate rinse }\end{array}$ \\
\hline Yoneyama (2002) & Japan & Nursing homes & 24 months & 366 & Professional care with povidone-iodine oral rinse & Usual oral care \\
\hline Juthani-Mehtha (2015) & USA & Nursing homes & 30 months & 575 & $\mathrm{CHX}$ oral rinse and enhanced oral care & Usual oral care \\
\hline \multicolumn{7}{|c|}{ Non-randomised controlled trials and other studies } \\
\hline McNally (2019) & USA & Controlled trial & 3.5 months & 2,890 & Enhanced oral care and cetylpyridinium oral rinse & Usual oral care \\
\hline Hollaar (2017) & Netherlands & Controlled trial & 12 months & 103 & Enhanced oral care and $\mathrm{CHX}$ oral rinse & Usual oral care \\
\hline Robertson (2013) & Canada & Quasi-experimental & 6 months & 83 & Enhanced oral care & Usual oral care \\
\hline Bassim (2008) & USA & Retrospective study & 79 weeks & 143 & Enhanced oral care & Usual oral care \\
\hline
\end{tabular}

Table 2 Forest plot of the RCTs. Review: enhanced oral hygiene maintenance for the prevention of non-ventilated pneumonia. Comparison: enhanced oral care versus usual care. Outcome: prevention of pneumonia

\begin{tabular}{|c|c|c|c|c|c|c|c|c|c|}
\hline \multirow[t]{2}{*}{ Study } & \multicolumn{2}{|c|}{ Experimental } & \multicolumn{2}{|c|}{ Control } & \multicolumn{2}{|l|}{ Risk ratio } & \multirow[t]{2}{*}{$\mathbf{R R}$} & \multirow[t]{2}{*}{$95 \% \mathrm{Cl}$} & \multirow[t]{2}{*}{ Weight } \\
\hline & Events & Total & Events & Total & & & & & \\
\hline Adachi (2002) & 5 & 40 & 9 & 48 & & & 0.67 & $(0.24 ; 1.83)$ & $8.5 \%$ \\
\hline Bourigault (2011) & 93 & 868 & 203 & 1,645 & 专 & & 0.87 & $(0.69 ; 1.09)$ & $29.9 \%$ \\
\hline Juthani-Mehtha (2015) & 119 & 277 & 94 & 298 & 1 & + & 1.36 & $(1.10 ; 1.69)$ & $30.5 \%$ \\
\hline Ohsawa (2003) & 5 & 25 & 6 & 24 & $\frac{1}{1}$ & & 0.80 & $(0.28 ; 2.28)$ & $8.0 \%$ \\
\hline Panchabhai (2009) & 2 & 136 & 4 & 164 & & & 0.60 & $(0.11 ; 3.24)$ & $3.6 \%$ \\
\hline Yoneyama (2002) & 21 & 184 & 34 & 182 & & & 0.61 & $(0.37 ; 1.01)$ & $19.6 \%$ \\
\hline Random effects model & 1,530 & & 2,361 & & & & 0.89 & $(0.64 ; 1.25)$ & $100.0 \%$ \\
\hline
\end{tabular}


using a meta-analysis strategy. Our findings in the effectiveness of enhanced oral care on preventing NVAP were null.

Most studies were performed in nursing home patients. While there are clearly substantive differences noted above between nursing home and acute care patients, they share the risk of acquired bacterial pneumonias for which the overall pathogenesis is similar. ${ }^{5}$ Thus, both patient cohorts serve as platforms upon which to assess the impact of procedures to reduce the oral bacterial burden as a mitigating strategy. Importantly, despite their dissimilarities, learnings from each group may be applicable and relevant to the other. Collectively, the PPA of enhanced oral hygiene failed to demonstrate a statistically significant impact on NVAP (pooled RR of 0.89, CI: 0.64-1.25, $\mathrm{p}$ value 0.50$)$ risk, which was diverse from that reported in an earlier meta-analysis of four RCTs (RR 0.61, CIs 0.40, 0.90, p value 0.02$).{ }^{33}$ When the evaluation was limited exclusively to nursing homes $(n=5)$ the impact of oral hygiene on NVAP RR was 0.90 , CIs $0.63,1.28, \mathrm{p}$ value 0.56 . It is noteworthy that enhanced oral hygiene negatively impacted risk in one United States study (RR of NVAP was 1.36). ${ }^{27}$ Aside from the patient population and location of

Table 3 Forest plot of the subgroup analysis of the RCTs. Review: enhanced oral hygiene maintenance for the prevention of nonventilated pneumonia. Comparison: chlorhexidine for oral care versus usual care. Outcome: prevention of pneumonia

\begin{tabular}{|c|c|c|c|c|c|c|c|c|}
\hline \multirow[t]{2}{*}{ Study } & \multicolumn{2}{|c|}{ Experimental } & \multicolumn{2}{|l|}{ Control } & \multirow[t]{2}{*}{ Risk ratio } & \multirow[t]{2}{*}{ RR } & \multirow[t]{2}{*}{$95 \% \mathrm{Cl}$} & \multirow[t]{2}{*}{ Weight } \\
\hline & Events & Total & Events & Total & & & & \\
\hline Bourigault (2011) & 93 & 868 & 203 & 1,645 & \multirow{2}{*}{+} & 0.87 & $(0.69 ; 1.09)$ & $46.8 \%$ \\
\hline Juthani-Mehtha (2015) & 119 & 277 & 94 & 298 & & 1.36 & $(1.10 ; 1.69)$ & $47.7 \%$ \\
\hline Panchabhai (2009) & 2 & 136 & 4 & 164 & & 0.60 & $(0.11 ; 3.24)$ & $5.5 \%$ \\
\hline Random effects model & \multicolumn{2}{|l|}{1,281} & \multicolumn{2}{|l|}{2,107} & & 1.05 & $(0.69 ; 1.60)$ & $100.0 \%$ \\
\hline
\end{tabular}

Heterogeneity: $1^{2}=77 \%, \operatorname{tau}^{2}=0.0830, p=0.01$

Table 4 Forest plot of the subgroup analysis of the RCTs. Review: enhanced oral hygiene maintenance for the prevention of nonventilated pneumonia. Comparison: professional dental care versus usual care. Outcome: prevention of pneumonia

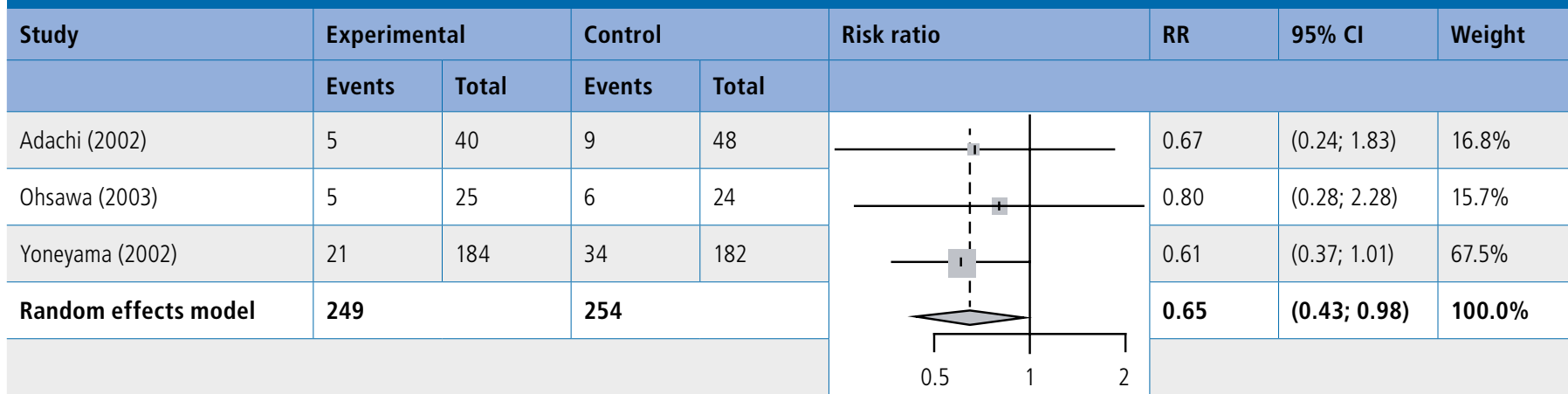

Heterogeneity: $\left.\right|^{2}=0 \%, \operatorname{tau}^{2}=0, p=0.90$

Table 5 Forest plot of the subgroup analysis of the RCTs. Review: enhanced oral hygiene maintenance for the prevention of nonventilated pneumonia. Comparison: enhanced oral care versus usual care. Outcome: prevention of mortality due to pneumonia

\begin{tabular}{|c|c|c|c|c|c|c|c|c|}
\hline \multirow[t]{2}{*}{ Study } & \multicolumn{2}{|c|}{ Experimental } & \multicolumn{2}{|l|}{ Control } & Risk ratio & \multirow[t]{2}{*}{ RR } & \multirow[t]{2}{*}{$95 \% \mathrm{Cl}$} & \multirow[t]{2}{*}{ Weight } \\
\hline & Events & Total & Events & Total & & & & \\
\hline Adachi (2002) & 2 & 40 & 8 & 48 & , it & 0.30 & $(0.07 ; 1.33)$ & $13.5 \%$ \\
\hline Bourigault (2011) & 15 & 868 & 26 & 1,645 & 11 & 1.09 & $(0.58 ; 2.05)$ & $26.6 \%$ \\
\hline Juthani-Mehtha (2015) & 122 & 277 & 88 & 298 & + & 1.49 & $(1.20 ; 1.86)$ & $32.7 \%$ \\
\hline Yoneyama (2002) & 14 & 184 & 30 & 182 & $T$ & 0.46 & $(0.25 ; 0.84)$ & $27.2 \%$ \\
\hline Random effects model & 1,369 & & 2,173 & & 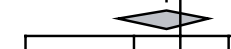 & 0.80 & $(0.40 ; 1.63)$ & $100.0 \%$ \\
\hline
\end{tabular}


Table 6 Forest plot of the non-RCTs (enhanced oral care in the prevention of NVAP). Review: enhanced oral hygiene maintenance for the prevention of non-ventilated pneumonia. Comparison: enhanced oral care versus usual care. Outcome: prevention of pneumonia

\begin{tabular}{|c|c|c|c|c|c|c|c|c|}
\hline \multirow[t]{2}{*}{ Study } & \multicolumn{2}{|c|}{ Experimental } & \multicolumn{2}{|l|}{ Control } & \multirow[t]{2}{*}{ Risk ratio } & \multirow[t]{2}{*}{ RR } & \multirow[t]{2}{*}{$95 \% \mathrm{Cl}$} & \multirow[t]{2}{*}{ Weight } \\
\hline & Events & Total & Events & Total & & & & \\
\hline McNally $(2019)^{28}$ & 25 & 1,403 & 26 & 1,487 & & 1.02 & $(0.59 ; 1.76)$ & $48.2 \%$ \\
\hline Hollaar $(2017)^{29}$ & 12 & 15 & 14 & 34 & & 1.94 & $(1.21 ; 3.12)$ & $51.8 \%$ \\
\hline Random effects model & 1,418 & & 1,521 & & & 1.42 & $(0.70 ; 2.88)$ & $100.0 \%$ \\
\hline
\end{tabular}

Heterogeneity: $I^{2}=74 \%, \operatorname{tau}^{2}=0.1921, p=0.05$

each trial, the intensity of the oral hygiene intervention was not uniform and varied principally in two ways: who performed the oral hygiene intervention and whether an antimicrobial rinse was included in the oral hygiene regimen.

The results of two non-RCTs support enhanced oral hygiene as an effective strategy to reduce NVAP risk, but in specific patient groups. A statistically significant reduction in NVAP incidence was reported in a neurosurgical patient cohort. ${ }^{30}$ Given the typical functional impediments associated with these patients, the finding is not surprising. A retrospective medical chart review in nursing home patients ${ }^{31}$ concluded that enhanced oral hygiene performed by a dedicated nursing assistant $(\mathrm{n}=78)$ significantly reduced NVAP outcomes compared to no oral care $(n=65)$.

Our subgroup analysis comparing the impact of healthcare provider credentials on outcomes showed that the standard of oral hygiene regimens $\mathrm{s}^{21,23,25}$ was enhanced for those in which dental professionals were involved, and these appeared to be more effective than those rendered by other providers in reducing NVAP risk (Table 4). This effect is comparable to previous metaanalysis. ${ }^{34}$ While this data supports the concept that effective oral microbial debridement favourably impacts NVAP risk, the conclusion that formal dental training results in demonstrably superior outcomes could be misleading as the effect might not be specifically attributed to variances in technical competencies, but rather to focus and time spent on the oral hygiene process. Whereas non-dental professionals typically number oral care as one of many patientrelated daily tasks, the sole emphasis of the dental professionals was on mouth hygiene. The observation that oral care delivered by a dedicated nursing assistant produced equivalent NVAP risk supports this argument. ${ }^{7}$ Competing time demands for services may limit nurses' capacity to deliver optimal mouthcare. ${ }^{28}$ Additional studies are necessary to more fully investigate the impact of provider qualifications on NVAP risk modification, since the cost implications of dedicated oral health aides, regardless of their qualification, is not trivial.

Two non-RCT studies in acute care hospital patients were informative. Out of 90 elderly patients admitted urgently for lower limb fractures, $10 \%$ of patients developed NVAP. ${ }^{10}$ While the authors found that pathogen colonisation of the mouth was higher in patients who developed NVAP, it was insufficient to explain differences between VAP and NVAP groups. Whereas NVAP risk was not associated with being dentate, tooth number, or heavy dental or denture plaque, it was associated with a specific bacterial carriage which the authors concluded was present before hospital admission.

In perhaps the largest study in an acute care hospital population, NVAP development was compared between patient self-brushing (control $n=1,487$ ) and enhanced nurse-delivered oral care (experimental $\mathrm{n}=1,403$; tooth brushing three times per day with a fresh toothbrush and daily use of an antiseptic rinse). Despite the designated oral care regimen, no impact on NVAP rates were seen between the control (1.7\%) and test groups (1.8\%). Critically, despite study-specific training and daily monitoring, nursing compliance was only 1.6 times per day, only slightly better than patient self-brushing frequency (1.2 times per day). However, when subjects from both arms were pooled and compared based on whether they developed NVAP, the odds ratio for NVAP decreased by $40 \%$ when tooth brushing increased by once per day, regardless of who performed the procedure. Importantly, this finding suggests that patient-directed education programmes and provision of oral care kits may be a valuable and cost-effective approach to NVAP control.

Given the challenges of cost and compliance with professionally delivered oral hygiene-based approaches, chemical disinfection offers a non-procedural alternative to reduce both the tooth-borne and mucosal oral bacterial burden. The latter clearly plays a role in VAP risk, and reduction of mucosal bacteria such as those residing on the tongue provides an important target for sustained antibacterial efficacy. ${ }^{35} \mathrm{CHX}$ remains the most popular agent for this purpose. However, in contrast to its reported efficacy in preventing VAP, its efficacy mitigating NVAP risk was inconsistent across the three RCTs (RR 1.05) we evaluated, ${ }^{22,25,27}$ perhaps because of differences in dose response effects and/or the impact of concurrent treatment. ${ }^{33}$

The contrasting efficacy of CHX rinses between NVAP and VAP is interesting. One might speculate the antimicrobial prophylaxis in the form of a topical agent is effective in preventing colonisation of the ventilator tubes in the same way that similar agents favourably impact cathetercentric infections. In the case of VAP, oral plaque accumulation could be exacerbated with placement of ventilation apparatus, especially in the premolar and molar areas. ${ }^{36}$ In non-ventilated patients, the microbiome and the environment is more fluid and subject to dilution effects of saliva, which might negatively impact efficacy. ${ }^{37,38}$ It is also possible that the time to onset which defines NVAP reduces the potential prophylactic 
efficacy of antimicrobials by compressing the time in which they might effectively impact NVAP outcomes.

Evaluation of the NVAP literature indicates that NVAP risk is not equivalent for all patients. ${ }^{39}$ High rates of NVAP are consistently noted in post-operative cancer patients, patients with neurological diseases and the elderly. The finding that dentate state (dentulous vs edentulous, and number of teeth) is not a clear risk determinant contradicts an oral hygiene strategy that focuses solely on tooth-borne bacteria. ${ }^{10}$ It is possible that a patient's oral health status may be a risk component to the extent that it reflects bacterial load. However, whether there is equivalent contribution to hospitalassociated pneumonia among the different microbiological eco-environments in the mouth (that is, tooth-borne bacteria vs mucosal bacterial niches like the dorsal tongue) is unclear. ${ }^{39,40}$ Likewise, the comparative effectiveness of different oral hygiene interventions on impacting bacterial pathogens is unresolved. ${ }^{41}$ Our analysis confirms the need for additional study to fully assess the benefit of OCI, optimise its timing and personalise the intensity of OCI-based individualising risk/benefit. It seems obvious that a 'one size fits all' approach for OCI would likely result in being excessive for many patients, but inadequate for others. Given the frequency and impact of NVAP, additional study is warranted.

\section{Conclusion}

In the introduction, we noted four questions to which answers would better define NVAP risk and intervention strategies. Given NVAP's potential clinical and economic burden, there is surprisingly little definitive documentation in the form of RCTs which speak to the efficacy of directed intervention methods. Most of the RCTs reported were done in nursing homes - most in Japan - and they conclude that structured enhanced oral hygiene regimens effectively reduced the rate of NVAP, and that enhanced oral hygiene delivered by dental professionals were most effective. Therefore, the generalisability of the results is limited. As a proof-of-concept, the results of such studies can be concluded to be positive with an overall reduction in VAP rates of greater than $10 \%$, but their broad translatability to the general hospital population is unclear. While good oral hygiene for hospitalised patients should be as consistent as handwashing and bathing, the current body of clinical research defining extended oral interventions as they relate to VAP risk, and the comparative effectiveness of various oral care interventions, is incomplete. Given the impact of NVAP, large, structured, randomised trials in which specific interventions are tested are critical.

\section{Acknowledgements}

This study was funded by an unrestricted grant from Sunstar to Primary Endpoint Solutions (SK, STS).

We thank Dr Dian Baker for her helpful comments during the preparation of this manuscript.

\section{Author contributions}

SK and STS: design, methods, data collections, analysis and preparation of paper. SP: analysis and preparation of paper. Sponsor's role in manuscript preparation: none.

\section{References}

1. Magill SS, O'Leary E, Janelle S J et al. Changes in Prevalence of Health Care-Associated Infections in U S. Hospitals. N Eng/ J Med 2018; 379: 1732-1744.

2. Davis J, Finley E. The breadth of hospital acquired pneumonia: Nonventilated versus ventilated patients in Pennsylvania. Penn Patient Safety Advis 2012; 9: 99-105.

3. Micek S T, Chew B, Hampton N, Kollef M H. A CaseControl Study Assessing the Impact of Nonventilated Hospital-Acquired Pneumonia on Patient Outcomes. Chest 2016; 150: 1008-1014.

4. Giuliano K K, Baker D, Quinn B. The epidemiology of nonventilator hospital-acquired pneumonia in the United States. Am J Infect Control 2018; 46: 322-327.

5. Raghavendran K, Mylotte J M, Scannapieco F A. Nursing home-associated pneumonia, hospitalacquired pneumonia and ventilator-associated pneumonia: the contribution of dental biofilms and periodontal inflammation. Periodontol 2000 2007: 44: 164-177.

6. Kalil A C, Metersky M L, Klompas M et al. Management of Adults with Hospital-acquired and Ventilator-associated Pneumonia: 2016 Clinical Practice Guidelines by the Infectious Diseases Society of America and the American Thoracic Society. Clin Infect Dis 2016; DOI: 10.1093/cid/ciw353.

7. Kelly A, Conell-Price J, Covinsky K et al. Length of stay for older adults residing in nursing homes at the end of life. J Am Geriatr Soc 2010; 58: 1701-1706.

8. Baker D, Quinn B. Hospital Acquired Pneumonia Prevention Initiative-2: Incidence of nonventilator hospital-acquired pneumonia in the United States. $A m$ J Infect Control 2018: 46: 2-7.

9. Azarpazhooh A, Leake J L. Systematic review of the association between respiratory diseases and oral health. J Periodontol 2006; 77: 1465-1482.

10. El-Solh A A, Pietrantoni C, Bhat A et al. Colonization of dental plaques: a reservoir of respiratory pathogens for hospital-acquired pneumonia in institutionalized elders. Chest 2004; 126: 1575-1582.

11. Moher D, Liberati A, Tetzlaff J, Altman D G, PRISMA Group. Preferred Reporting Items for Systematic Reviews and Meta-Analyses: The PRISMA Statement. PLoS Med 2009; DOI: 10.1371/journal.pmed.1000097.

12. Deeks J J, Dinnes J, D'Amico R et al. Evaluating nonrandomised intervention studies. Health Technol Assess 2003; 7: 1-173.

13. Higgins J P, Altman D G, Gøtzsche P C et al. The Cochrane Collaboration's tool for assessing risk of bias in randomised trials. BMJ 2011; DOI: 10.1136/ bmj.d5928.

14. DerSimonian R, Laird N. Meta-analysis in clinical trials. Control Clin Trials 1986; 7: 177-188.

15. Cochran W G. The comparison of percentages in matched samples. Biometrika 1950; 37(3-4): 256-266.

16. IntHout J, loannidis J P, Borm G F. The HartungKnappSidik-Jonkman method for random effects meta-analysis is straightforward and considerably outperforms the standard DerSimonianLaird method. BMC Med Res Methodol 2014; 14: 25.

17. Mantel N, Haenszel W. Statistical aspects of the analysis of data from retrospective studies of disease. J Natl Cancer Inst 1959; 22: 719-748.

18. Böhning D, Malzahn U, Dietz E, Schlattmann P, Viwatwongkasem C, Biggeri A. Some general points in estimating heterogeneity variance with the DerSimonian-Laird estimator. Biostatistics 2002; 3: 445-457.

19. Sánchez-Meca J, Marín-Martínez F. Confidence intervals for the overall effect size in randomeffects meta-analysis. Psychol Methods 2008; 13: 31-48.

20. Sidik K, Jonkman J N. Simple heterogeneity variance estimation for meta-analysis. J Roy Stat Soc 2005; 54: 367-384.

21. Adachi M, Ishihara K, Abe S, Okuda K, Ishikawa T. Effect of professional oral health care on the elderly living in nursing homes. Oral Surg Oral Med Oral Pathol Oral Radiol Endod 2002; 94: 191-195.

22. Bourigault C, Lietard C, Golmard J L et al. Impact of buccodental healthcare on the prevention of pneumonia in geriatrics: a cluster-randomised trial. J Hosp Infect 2011; 77: 78-80.

23. Ohsawa T, Yoneyama T, Hashimoto K, Kubota E, Ito M, Yoshida K. Effects of professional oral health care on the ADL of elderly patients in a nursing home. Bulletin Kanagawa Dent College 2003; 31: 51-54.

24. Lam O L, McMillan A S, Samaranayake L P, Li L S, McGrath C. Randomized clinical trial of oral health promotion interventions among patients following stroke. Arch Phys Med Rehabil 2013; 94: 435-443.

25. Panchabhai T S, Dangayach N S, Krishnan A, Kothari V M, Karnad D R. Oropharyngeal cleansing with $0.2 \%$ chlorhexidine for prevention of nosocomial pneumonia in critically ill patients: an open-label randomized trial with $0.01 \%$ potassium permanganate as control. Chest 2009; 135: 1150-1156.

26. Yoneyama T, Yoshida M, Ohrui T et al. Oral care reduces pneumonia in older patients in nursing homes. J Am Geriatr Soc 2002; 50: 430-433.

27. Juthani-Mehta M, Van Ness P H, McGloin J et al. A cluster-randomized controlled trial of a multicomponent intervention protocol for pneumonia prevention among nursing home elders. Clin Infect Dis 2015; 60: 849-857.

28. McNally E, Krisciunas G P, Langmore S E, Crimlisk J T, Pisegna J M, Massaro J. Oral Care Clinical Trial to Reduce Non-Intensive Care Unit, Hospital-Acquired Pneumonia: Lessons for Future Research. J Healthcare Qual 2019; 41: 1-9.

29. Hollaar $V R$ Y , van der Putten $G J$, van der Maarel-Wierink C D, Bronkhorst E M, de Swart B J M, Creugers N H J. The effect of a daily application of a $0.05 \%$ chlorhexidine oral rinse solution on the incidence of aspiration pneumonia in nursing home residents: a multicentre study. BMC Geriatr 2017 17: 128.

30. Robertson T, Carter D. Oral intensity: reducing nonventilatorassociated hospitalacquired pneumonia in care-dependent, neurologically impaired patients. Can J Neurosci Nurs 2013; 35: 10-17.

31. Bassim C W, Gibson G, Ward T, Paphides B $M$, Denucci D J. Modification of the risk of mortality from pneumonia with oral hygiene care. J Am Geriatr Soc 2008; 56: 1601-1607.

32. Ranganathan P, Pramesh C S, Aggarwal R. Common pitfalls in statistical analysis: Measures of agreement Perspect Clin Res 2017; 8: 187-191.

33. Tantipong $\mathrm{H}$, Morkchareonpong $\mathrm{C}$, Jaiyindee S, Thamlikitkul V. Randomized controlled trial and metaanalysis of oral decontamination with $2 \%$ chlorhexidine solution for the prevention of 
34. Kaneoka A, Pisegna J M, Miloro K V et al. Prevention of Healthcare-Associated Pneumonia with Oral Care in Individuals Without Mechanical Ventilation: A Systematic Review and Meta-Analysis of Randomized Controlled Trials. Infect Control Hosp Epidemiol 2015; 36: 899-906.

35. Bordas A, McNab R, Staples A M, Bowman J, Kanapka J, Bosma M P. Impact of different tongue cleaning methods on the bacterial load of the tongue dorsum. Arch Oral Biol 2008; DOI: 10.1016/ S0003-9969(08)70004-9.

36. Jones D J, Munro C L, Grap M J. Natural history of dental plaque accumulation in mechanically ventilated adults: a descriptive correlational study. Intensive Crit Care Nurs 2011; 27: 299-304.

37. Ito M, Kawakami M, Ohara E, Muraoka K, Liu M. Predictors for achieving oral intake in older patients with aspiration pneumonia: Videofluoroscopic evaluation of swallowing function. Geriatr Gerontol Int 2018; 18: 1469-1473.

38. Estes R J, Meduri G U. The pathogenesis of ventilatorassociated pneumonia: I. Mechanisms of bacterial transcolonization and airway inoculation. Intensive Care Med 1995; 21: 365-383.

39. Di Pasquale M, Aliberti S, Mantero M, Bianchini S, Blasi F. Non-Intensive Care Unit Acquired Pneumonia: A New Clinical Entity? Int J Mol Sci 2016: 17: 287.

40. Hong C, Aung M M, Kanagasabai K, Lim C A, Liang $S$, Tan K S. The association between oral health status and respiratory pathogen colonization with pneumonia risk in institutionalized adults. Int I Dent Hyg 2018; DOI: 10.1111/idh.12321.

41. Lam O L, McGrath C, Li L S, Samaranayake L P. Effectiveness of oral hygiene interventions against oral and oropharyngeal reservoirs of aerobic and facultatively anaerobic gram-negative bacilli. $A m$ J Infect Control 2012; 40: 175-182.

\begin{tabular}{|c|c|}
\hline \multirow{4}{*}{$\begin{array}{l}\text { Search in PubMed } \\
\text { (inception until January 2019) }\end{array}$} & $\begin{array}{l}\text { Nursing homes [Title/Abstract] OR intermediate care } \\
\text { facilities [Title/Abstract] OR nursing facilities [Title/Abstract] } \\
\text { AND elderly care [Title/Abstract] }\end{array}$ \\
\hline & $\begin{array}{l}\text { Oral hygiene [Title/Abstract] OR mouth care [Title/Abstract] } \\
\text { OR mouthwash [Title/Abstract] OR oral chlorhexidine [Title/ } \\
\text { Abstract] OR oral povidone-iodine [Title/Abstract] OR tooth } \\
\text { brushing [Title/Abstract] OR dental care [Title/Abstract] } \\
\text { OR oral antiseptic [Title/Abstract] OR oral rinse [Title/Abstract] }\end{array}$ \\
\hline & $\begin{array}{l}\text { Pneumonia [Title/Abstract] OR lung infection [Title/Abstract] } \\
\text { OR non-ventilator pneumonia [Title/Abstract] OR respiratory } \\
\text { tract diseases [Title/Abstract] OR lung diseases [Title/ } \\
\text { Abstract] OR hospital-acquired pneumonia [Title/Abstract] } \\
\text { OR nosocomial pneumonia [Title/Abstract] }\end{array}$ \\
\hline & $\begin{array}{l}\text { Cross infection [Title/Abstract] OR nosocomial infection [Title/ } \\
\text { Abstract] OR infection, hospital [Title/Abstract] OR health care } \\
\text { associated infections [Title/Abstract] }\end{array}$ \\
\hline \multirow{5}{*}{$\begin{array}{l}\text { Search in Embase } \\
\text { (from } 1 \text { January } 1990 \text { to January 2019) }\end{array}$} & $\begin{array}{l}\text { 'cross infection':ab,ti OR 'nosocomial infection':ab,ti } \\
\text { OR 'infection, hospital':ab,ti OR 'health care associated } \\
\text { infections':ab,ti) AND [1990-2019]/py }\end{array}$ \\
\hline & $\begin{array}{l}\text { ('nursing homes':ab,ti OR 'intermediate care facilities':ab,ti } \\
\text { OR 'nursing facilities':ab,ti OR 'elderly care':ab,ti) AND } \\
\text { [1990-2019]/py }\end{array}$ \\
\hline & $\begin{array}{l}\text { ('oral hygiene':ab,ti OR 'mouth care':ab,ti OR 'oral } \\
\text { chlorhexidine':ab,ti OR 'oral povidone-iodine':ab,ti OR 'tooth } \\
\text { brushing':ab,ti OR 'dental care':ab,ti OR 'oral antiseptic':ab,ti } \\
\text { OR 'oral rinse':ab,ti) AND [1990-2019]/py }\end{array}$ \\
\hline & $\begin{array}{l}\text { ('pneumonia':ab,ti OR 'lung infection':ab,ti OR 'non-ventilator } \\
\text { pneumonia':ab,ti OR 'respiratory tract diseases':ab,ti } \\
\text { OR 'hospital-acquired pneumonia':ab,ti OR 'nosocomial } \\
\text { pneumonia':ab,ti) AND [1990-2019]/py }\end{array}$ \\
\hline & 1 AND 2 AND 3 AND 4 \\
\hline
\end{tabular}

